\title{
Protein Microgels from Amyloid Fibril Networks
}

Ulyana Shimanovich, ${ }^{1}$ Igor Efimov, ${ }^{1}$ Thomas O. Mason, ${ }^{1}$ Patrick Flagmeier, ${ }^{1}$ Alexander K. Buell, ${ }^{1}$

Aharon Gedanken, ${ }^{2}$ Sara Linse, ${ }^{3}$ Karin S. Akerfeldt,${ }^{4}$ Christopher M. Dobson, ${ }^{* 1}$ David A. Weitz, ${ }^{* 5}$ and Tuomas P. J. Knowles ${ }^{* 1}$

1 Department of Chemistry, University of Cambridge, Lensfield Rd, Cambridge, CB21EW, UK.

2 Department of Chemistry, Bar-Ilan University, Ramat-Gan, 52900, Israel.

3 Department of Biochemistry and Structural Biology, Lund University, Lund, 22100, Sweden.

4 Department of Chemistry, Haverford College, Haverford, PA 19041, USA.

5 Department of Physics and School of Engineering and Applied Science, Harvard University, 29 Oxford Street, Cambridge, MA 02138.USA

\section{SUPPLEMENTARY INFORMATION}

The $\mathrm{pH}$ dependence of the release kinetics was probed on ThT and penicillin $\mathrm{V}$ molecules as a function of time (Fig. S2 a and b). Our choice of ThT and penicillin drug-like molecules was based on the slowest and fastest release profiles, respectively, obtained previously (Fig. 3d) for neutral $\mathrm{pH}$. 
a
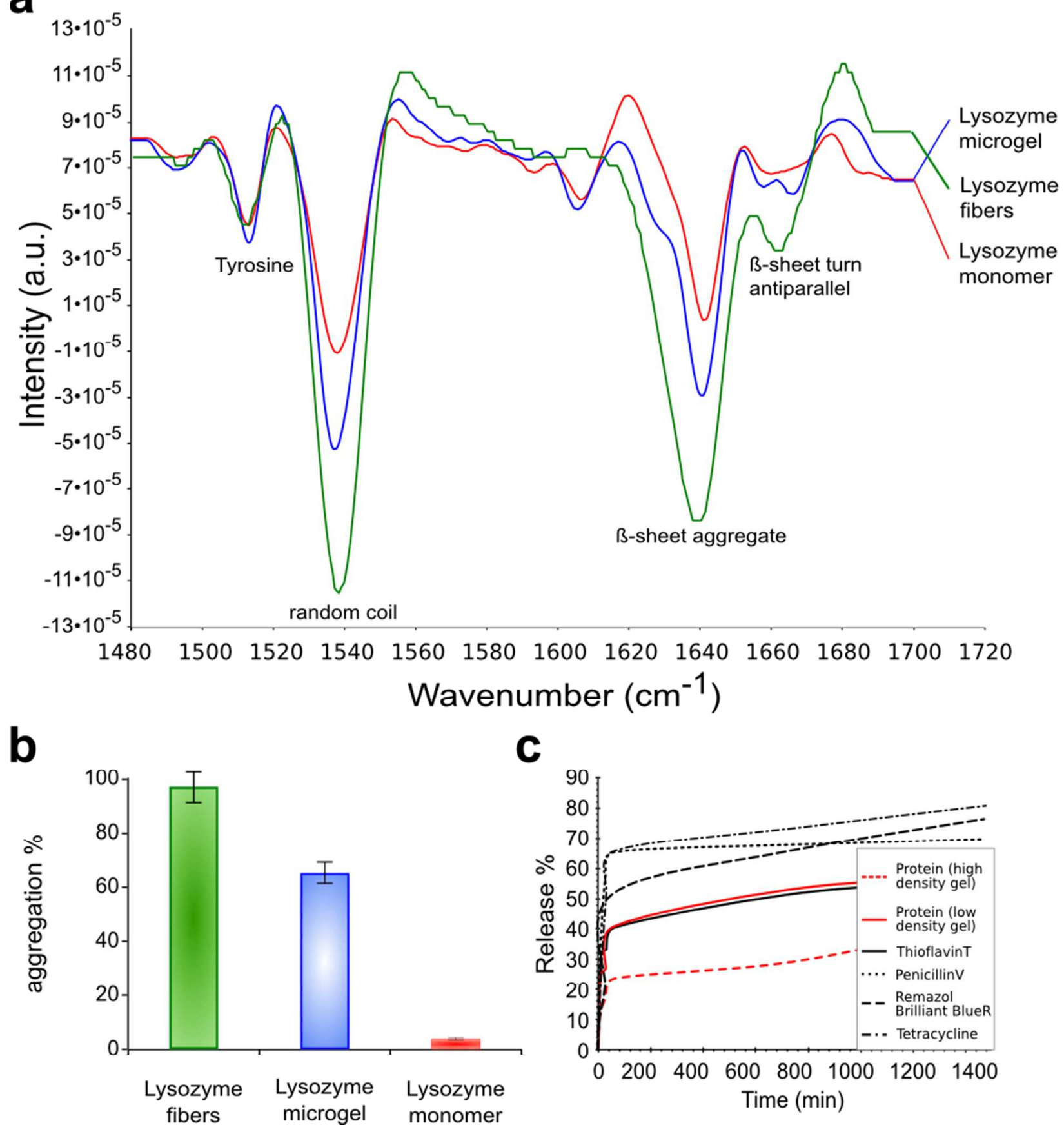

Figure S1. (a) FTIR spectra of lysozyme monomers, lysozyme fibbers and lysozyme microgel; (b) chart summarizing $\beta$-sheet content (in \%) in lysozyme microgels; (c) graph summarising release kinetics in $\boldsymbol{\alpha}$-MEM media as a function of time. 
a

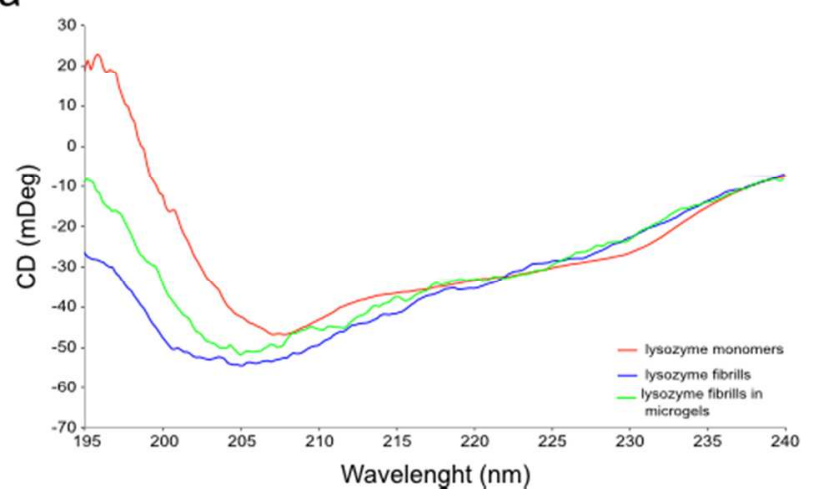

b

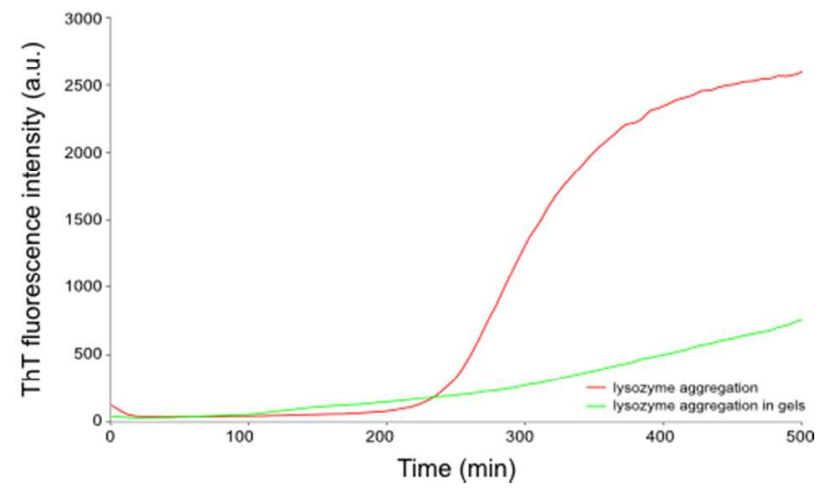

Figure S2. (a) Circular Dichroism (CD) spectra of lysozyme, indicating significant $\beta$-sheet content in its monomeric and nanofibrillar states in microgel capsules.; (b) Lysozyme aggregation kinetics followed by ThT fluorescence intensity as a function of time.
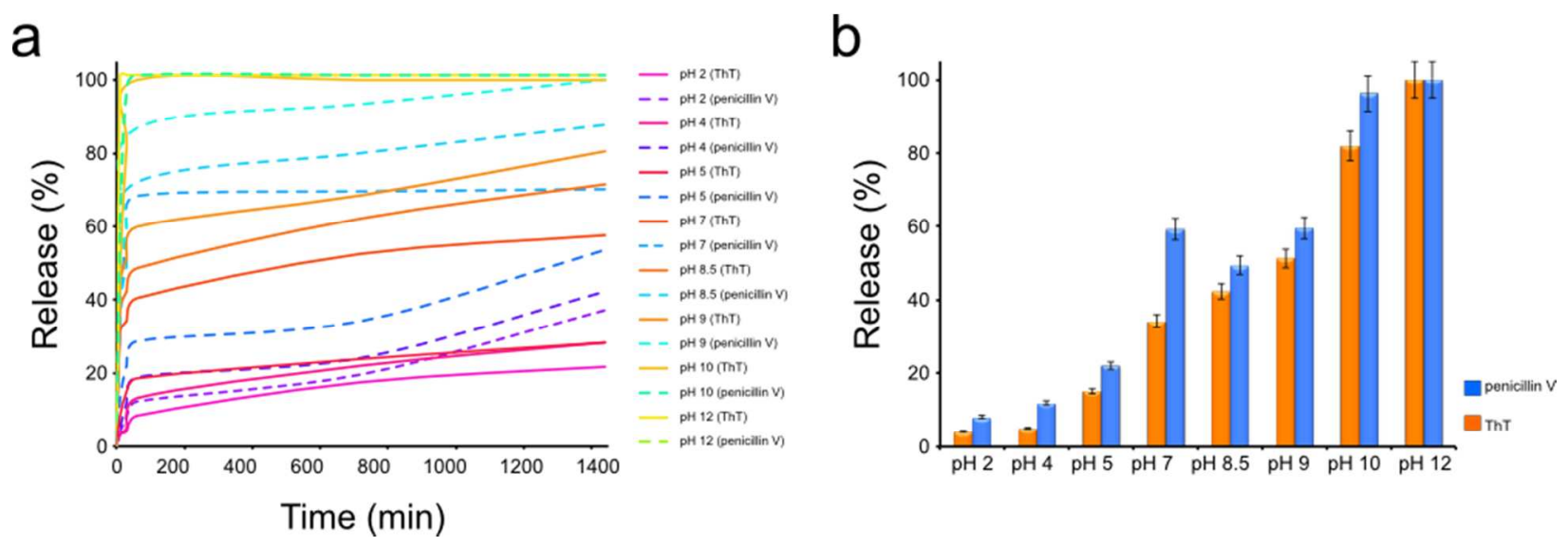

Figure S3. (a) pH-dependence of the release kinetics for ThT and penicillin V molecules.; (b) Released content (in \% after $30 \mathrm{~min}$ ) for ThT and penicillin $\mathrm{V}$ molecules at different $\mathrm{pH}$ values. 


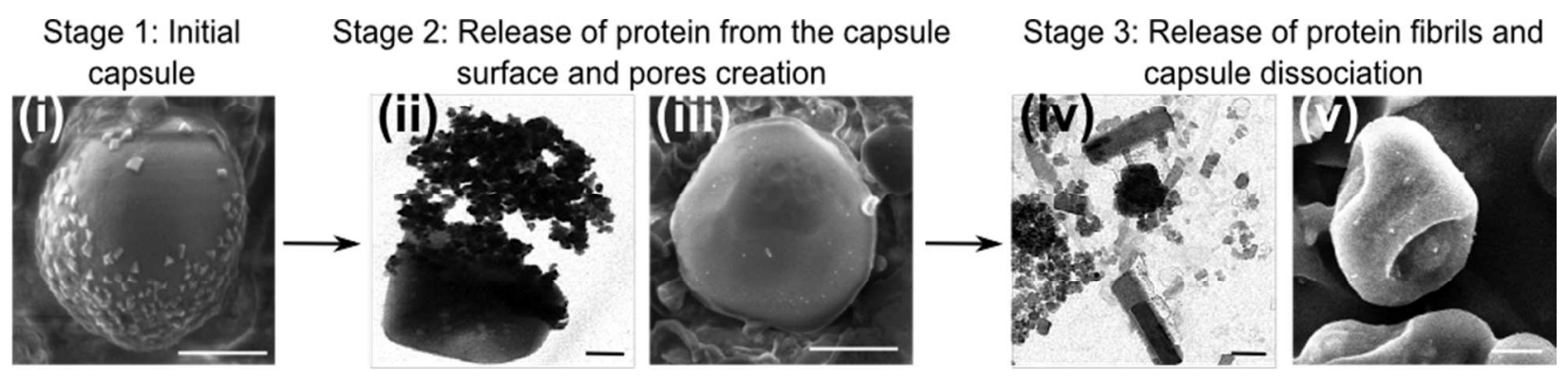

Figure S4. Schematic representation of the release mechanism for lysozyme capsules. (i) SEM image of a lysozyme microgel capsule, (ii) TEM image of a lysozyme microgel with protein layer detached from the surface. Scale bar $=1 \mu \mathrm{m}$ (iii) SEM image of the microgel illustrating the creation of the pores on the surface. (iv) TEM image of lysozyme fibrils released from the microgel. Scale Bar $=0.1 \mu \mathrm{m}$. (v) SEM images of disaggregated lysozyme capsule: scale bars for SEM images $=10 \mu \mathrm{m}$.

a

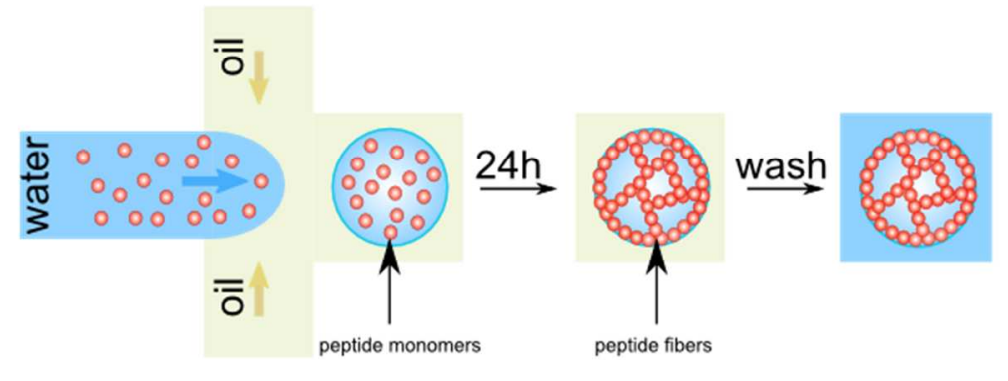

b

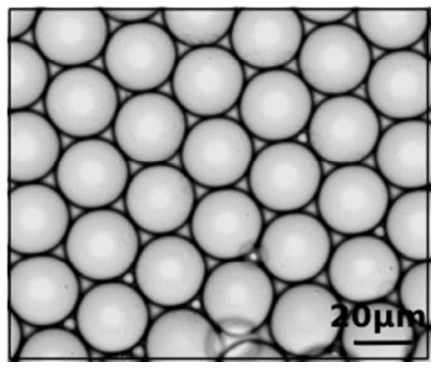

Figure S5. (a) Schematic representation of the KD peptide microgel synthesis; (b) microscopy image of nanofibrilar microgels formed from the KD peptide. Scale Bar $=20 \mu \mathrm{m}$.

\section{Corresponding Author}

*E-mail:cmd44@cam.ac.uk; weitz@seas.harvard.edu; tpjk2@cam.ac.uk 